\title{
Erratum: Tree mortality: Large trees losing out to drought
}

Michael G. Ryan

Nature Plants 1, 15150 (2015); published online 6 October 2015; corrected 9 October 2015

In the original version of this News and Views, the caption of the photograph was incorrect. The trees shown are piñon pines (Pinus edulis) in the Jemez Mountains of New Mexico, USA. This has been corrected. 